\title{
Screening for Growth Hormone Deficiency in Three-year-old Children
}

\author{
Shigeki Miyamoto, Nozomu Sasaki and Hiroshi Ikegami \\ Division of Endocrinology, Chiba Children's Hospital (S.M., N.S.), \\ Chiba City Public Health Center (H.I.), Chiba, Japan
}

\begin{abstract}
We attempted to clarify the efficacy of GH treatment in three-year-old children with GH deficiency. Over 10,000 children aged three were evaluated for their height SD scores at public health centers during 1990 and 1991. Children with height SD scores of less than - 2SD were advised to visit children's hospitals. GH secretion was assessed in 19 children by the responses to insulin and arginine, and by GH levels during three hours' sleep. GH treatment was applied in 16 children based on the criteria of the Foundation of Growth Science in Japan. Eight children were treated with hGH for one year. Their height SD scores improved from $-3.14 \pm 0.83$ to $-2.17 \pm$ $0.69(\mathrm{p}<0.001)$.

The results did not show the frequency of GH deficiency among three-year-old children, but of those who were found and treated, their height SD scores improved. Longer-term studies may clarify the benefit of $\mathrm{GH}$ treatment initiated at an early age.
\end{abstract}

Key words: GH deficiency, screening

\section{Introduction}

Therapeutic options have increased for short children through the commercial availability of biosynthetic hGH. Though hGH treatment may be more effective for achieving normal height when used in early childhood, there is no generally accepted method for the early detection of GH-deficient children.

We screened short children at their three year medical check and evaluated GH secretion according to the criteria of the Foundation of Growth Science in Japan [1].

Correspondence: Shigeki Miyamoto, M.D., 579-1, Heta-Cho, Midori-ku, Chiba 266 Japan

\section{Subjects and Methods}

The subjects were children who visited a Public Health Center for a three year medical check-up during 1990 and 1991. Children whose height was below $-2 \mathrm{SD}$ of the mean were advised to visit children's hospitals. At the first visit, complete blood count, blood chemistry, urinalysis, F-T4, TSH and IGF-I levels were examined.

After confirming normal thyroid function, GH secretion was assessed by insulin $(0.1 \mathrm{U} / \mathrm{kg}$ iv) and arginine $(0.5 \mathrm{~g} / \mathrm{kg}$ iv) loading tests (criterion 1), and by the mean GH levels during the first three hours of sleep (criterion 2). 
Patients were treated with biosynthetic recombinant $\mathrm{hGH}$ at a total of $0.5 \mathrm{U} /$ week, given by five to seven divided sc injections.

\section{Results}

GH secretion was examined in 19 out of 24 children who visited the hospital. Table 1 shows the clinical data. Their height SD score was $-3.00 \pm 0.57$ (mean $\pm 1 \mathrm{SD}$ ), and the range was -2.24 to -4.42 . In all cases both

Table 1. Patient data

\begin{tabular}{cccccccc}
\hline No. & sex & \multicolumn{2}{c}{ Age } & Height(H) & SD & Father's H Mother's H \\
\hline 1 & $\mathrm{~F}$ & $3 \mathrm{yr}$ & $3 \mathrm{mo}$ & $84.0 \mathrm{~cm}$ & -3.80 & $172 \mathrm{~cm}$ & $152 \mathrm{~cm}$ \\
2 & $\mathrm{M}$ & 3 & 3 & 88.5 & -2.31 & 156 & 153 \\
3 & $\mathrm{~F}$ & 3 & 3 & 86.3 & -2.81 & 169 & 145 \\
4 & $\mathrm{~F}$ & 3 & 3 & 87.1 & -2.54 & 171 & 150 \\
5 & $\mathrm{M}$ & 3 & 5 & 89.2 & -2.38 & 167 & 162 \\
6 & $\mathrm{M}$ & 3 & 3 & 86.3 & -3.01 & 172 & 156 \\
7 & $\mathrm{M}$ & 3 & 4 & 82.3 & -4.42 & 162 & 153 \\
8 & $\mathrm{~F}$ & 3 & 10 & 90.2 & -2.69 & 165 & 157 \\
9 & $\mathrm{~F}$ & 3 & 3 & 86.7 & -2.64 & 165 & 153 \\
10 & $\mathrm{M}$ & 3 & 3 & 86.1 & -3.07 & 161 & 151 \\
11 & $\mathrm{M}$ & 3 & 3 & 84.6 & -3.54 & 163 & 148 \\
12 & $\mathrm{~F}$ & 3 & 3 & 86.7 & -3.26 & 166 & 153 \\
13 & $\mathrm{M}$ & 3 & 6 & 85.5 & -3.57 & 168 & 154 \\
14 & $\mathrm{~F}$ & 3 & 4 & 85.8 & -3.14 & 170 & 143 \\
15 & $\mathrm{~F}$ & 3 & 4 & 88.4 & -2.29 & 160 & 155 \\
16 & $\mathrm{~F}$ & 3 & 11 & 92.2 & -2.24 & 167 & 148 \\
17 & $\mathrm{M}$ & 3 & 3 & 85.6 & -3.07 & 172 & 145 \\
18 & $\mathrm{~F}$ & 3 & 4 & 85.8 & -3.14 & 167 & 148 \\
19 & $\mathrm{~F}$ & 3 & 2 & 85.0 & -3.07 & 172 & 151 \\
\hline mean & 3 & 4 & & -3.00 & 167 & 151 \\
SD & & & & & 0.57 & 5 & 5 \\
\hline
\end{tabular}

Table 2. Criterion for GH Therapy (1)

\begin{tabular}{ccccc}
\hline No. & \multicolumn{2}{c}{$\mathrm{GH}$ (peak) } & score & total \\
& insulin & arginine & & \\
\hline 1 & $1.7 \mathrm{ng} / \mathrm{ml}$ & $3.3 \mathrm{ng} / \mathrm{ml}$ & $4+4$ & 8 \\
2 & 6.1 & 5.3 & $4+4$ & 8 \\
3 & 1.8 & 5.5 & $4+4$ & 8 \\
4 & 4.0 & 6.7 & $4+4$ & 8 \\
5 & 4.8 & 7.4 & $4+3$ & 7 \\
6 & 7.5 & 7.3 & $3+3$ & 6 \\
7 & 8.6 & 7.5 & $3+3$ & 6 \\
8 & 9.2 & 7.2 & $3+3$ & 6 \\
9 & 8.2 & 8.4 & $3+3$ & 6 \\
10 & 8.2 & 7.7 & $3+3$ & 6 \\
11 & 8.1 & 7.7 & $3+3$ & 6 \\
12 & 8.2 & 8.4 & $3+3$ & 6 \\
13 & 21.0 & 8.6 & $0+3$ & 3 \\
14 & 10.4 & 2.2 & $0+4$ & 4 \\
15 & 16.0 & 3.6 & $0+4$ & 4 \\
16 & 12.5 & 2.9 & $0+4$ & 4 \\
17 & 17.0 & 3.2 & $0+4$ & 4 \\
18 & 13.1 & 9.7 & $0+3$ & 3 \\
19 & 10.6 & 10.7 & $0+0$ & 0 \\
\hline
\end{tabular}

parents were shorter than the average adult Japanese height.

Table 2 shows the peak GH levels after pharmacologic provocation tests. Four patients showed complete GH deficiency and eight had partial deficiency. Table 3 shows the results as defined by criterion 2 . One patient showed neurosecretary dysfunction. A summary of the criteria is shown in Table 4. HGH treatment was started in 16 out of 19 short children, of whom eight have already been under treatment for one year. Their

Table 3. Criterion for GH Therapy (2)

\begin{tabular}{|c|c|c|c|c|c|c|}
\hline No. & $\begin{array}{l}\text { Perinatal } \\
\text { Abnormality }\end{array}$ & $\begin{array}{l}\text { nean GH (sleep) } \\
(\mathrm{ng} / \mathrm{ml})\end{array}$ & $\begin{array}{c}\text { urine } \mathrm{GH} \\
(\mathrm{pg} / \mathrm{mgCr})\end{array}$ & $\begin{array}{c}\text { IGF-I } \\
(\mathrm{U} / \mathrm{ml}, \mathrm{ng} / \mathrm{ml} *)\end{array}$ & $\begin{array}{c}\mathrm{BA} / \mathrm{CA} \\
\% \\
\end{array}$ & score \\
\hline 1 & $+(1)$ & $1.8(3)$ & ND & 0.24 & 31(1) & 5 \\
\hline 2 & $-(0)$ & $4.1(3)$ & ND & 0.38 & $82(0)$ & 3 \\
\hline 3 & $-(0)$ & $3.2(3)$ & $27(0)$ & 0.26 & $59(1)$ & 4 \\
\hline 4 & $-(0)$ & $3.0(3)$ & $4(1)$ & 0.42 & $47(1)$ & 5 \\
\hline 5 & $-(0)$ & $4.2(2)$ & $27(0)$ & 1.32 & $62(1)$ & 3 \\
\hline 6 & $-(0)$ & $4.2(2)$ & ND & 0.19 & $46(1)$ & 3 \\
\hline 7 & $-(0)$ & $3.5(3)$ & ND & 0.11 & $45(1)$ & 4 \\
\hline 8 & $-(0)$ & $6.3(0)$ & $33(0)$ & 0.51 & $49(1)$ & 1 \\
\hline 9 & $-(0)$ & $5.3(0)$ & $15(0)$ & 0.84 & 46(1) & 1 \\
\hline 10 & $-(0)$ & $2.8(3)$ & ND & 0.41 & $38(1)$ & 4 \\
\hline 11 & $-(0)$ & $5.4(0)$ & ND & 0.47 & $46(1)$ & 1 \\
\hline 12 & $-(0)$ & $5.1(0)$ & $46(0)$ & 0.84 & $46(1)$ & 1 \\
\hline 13 & $+(1)$ & $3.1(3)$ & ND & 0.78 & $64(1)$ & 5 \\
\hline 14 & $-(0)$ & $3.1(3)$ & ND & 0.21 & $60(1)$ & 4 \\
\hline 15 & $-(0)$ & $4.0(3)$ & $12(0)$ & 0.77 & $60(1)$ & 4 \\
\hline 16 & $-(0)$ & $4.6(2)$ & $29(0)$ & $62.1^{*}$ & $65(1)$ & 3 \\
\hline 17 & $-(0)$ & $5.6(0)$ & $\mathrm{ND}$ & 0.89 & $39(1)$ & 1 \\
\hline 18 & $-(0)$ & $7.0(0)$ & $40(0)$ & 0.48 & $69(1)$ & 1 \\
\hline 19 & $-(0)$ & $2.8(3)$ & $36(0)$ & $79.0 *$ & $55(1)$ & 4 \\
\hline
\end{tabular}

ND: not done.

Table 4. Criteria for GH Therapy

\begin{tabular}{cccl}
\hline No. & Criterion(1) & Criterion(2) & Evaluation \\
\hline 1 & 8 & 5 & therapy \\
2 & 8 & 3 & therapy \\
3 & 8 & 3 & therapy \\
4 & 8 & 5 & therapy \\
5 & 7 & 4 & therapy \\
6 & 6 & 3 & therapy \\
7 & 6 & 4 & therapy \\
8 & 6 & 1 & therapy \\
9 & 6 & 1 & therapy \\
10 & 6 & 4 & therapy \\
11 & 6 & 1 & therapy \\
12 & 6 & 1 & therapy \\
13 & 3 & 5 & therapy \\
14 & 4 & 4 & therapy (6mo) \\
15 & 4 & 4 & therapy (6mo) \\
16 & 4 & 3 & therapy $6 \mathrm{mo})$ \\
17 & 4 & 1 & follow-up \\
18 & 3 & 1 & follow-up \\
19 & 0 & 4 & follow-up \\
\hline
\end{tabular}




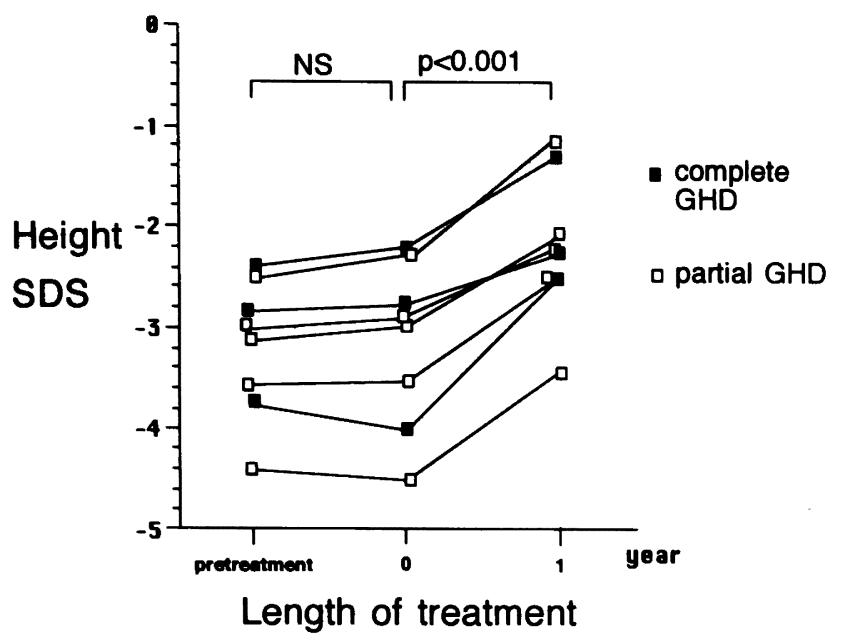

Fig 1. Height SD score changes after 1 year of hGH treatment.

height SD scores were significantly improved from -3.14 to $-2.17 \quad(\mathrm{p}<0.001) \quad$ (Fig.1). There was no significant difference in improvement of the height SD scores between patients with complete or partial GH deficiency.

\section{Discussion}

$\mathrm{HGH}$ is now easily obtainable because of advances in biosynthetic techniques. Several studies have documented the beneficial effects of hGH treatment in short children who do not meet the criteria for GH deficiency [2]. This raises the question: is the early diagnosis of GH deficiency important? Screening for GH deficiency was performed at the age of three; of 24 short children who were investigated, 16 were found to be $\mathrm{GH}$-deficient; $\mathrm{HGH}$ therapy proved effective for these patients. Josefsberg et al. [3] reported a greater effect of $\mathrm{hGH}$ therapy in children below five years of age. There are few reports about the early detection of GH deficiency, and we attempted to evaluate a screening program for this. Our results also showed an early improvement of height SD scores within one year of treatment. Longer-term experience may confirm the usefulness of the three year medical check at public health centers.

\section{References}

1. The Foundation of Growth Science in Japan. Indication of hGH treatment. 1991.

2. Moore WV, Moore KC, Gifford R, Hollowell JG, Donaldson DL. Long-term treatment with growth hormone of children with short stature and normal growth hormone secretion. J Pediatr 1992; 120: 702-8.

3. Josefsberg Z, Bauman B, Perzelan A, Laron Z. Greater efficiency of human growth hormone therapy in children below five years of age with growth hormone deficiency - a five-year follow-up study - . Hormone Res. 1987; 27: 126-33. 